\title{
Electron beam radiotherapy in giant non- melanoma skin cancers of the head in elderly inoperable patients: a single centre retrospective study
}

\begin{abstract}
Background: Electron beam three-dimensional conformal radiotherapy (3D-CRT) may be an alternative effective option for the treatment of giant nonmelanoma skin cancers (NMSCs) of the head in older patients unfit for surgery. Here, we present a retrospective study of older patients with giant NMSCs of the head treated with 3D-conformal electron beam radiotherapy, including analysis of clinical outcomes and factors impacting local control.
\end{abstract}

Materials and methods: Fifty older patients with sixty T3-T4 giant and aggressive NMSC lesions of the head, irradiated between 2005 and 2015, were retrospectively studied. Treatment consisted of one or two fields of customized electron beams planned using 3D conformal radiotherapy. Univariate analysis with Chi-square, Cox proportional hazard ratio, and Pearson's covariance for multivariate analyses, assuming $\mathrm{P}<0.05$ for statistical significance, were used to assess the impact of clinical and dosimetric parameters on local control.

Results: Complete response (CR) occurred in 15 patients and 25 lesions. Bleeding control was obtained for all symptomatic lesions. Cumulative local progression free survival rates at 2 and 5 years were $85 \%$ and $78 \%$, respectively. On univariate analysis several factors were found to significantly impact local progression, including diabetes $(\mathrm{P}<0.001)$ and primary site $(\mathrm{P}<0.03)$, with scalp being significant for durable local control $(\mathrm{P}<0.02)$. On multivariate analysis, both factors were confirmed as significant prognostic indicators of local control. Treatment was well-tolerated with acceptable acute and late toxicity and good palliation effect.

Conclusion: Customized electron beam 3D CRT for giant NMSCs of the head not amendable to surgery in frail older patients can be considered an effective and safe option in both curative and palliative settings.

Keywords: elderly, basal skin cancer, squamous skin cancer, electron beam, scalp
Volume II Issue 4 - 2020

\author{
Grazia Lazzari,' Perri Francesco, ${ }^{2}$ Giuseppina \\ Scarpati della Vittoria, ${ }^{3}$ Maria Giovanna \\ Monis,' Giovanna Porrazzo,' Giovanni \\ Silvano' \\ 'Radiation Oncology Unit, San Giuseppe Moscati Hospital, \\ Taranto, Italy \\ 2Medical and Experimental Head and Neck Oncology, National \\ Cancer Institute Fondazione Pascale, Napoli, Italy \\ ${ }^{3}$ Medical Oncology Unit, San Rocco Hospital, Sessa Aurunca, \\ Caserta, Italy
}

Correspondence: Grazia Lazzari, Radiation Oncologist, Radiation Oncology Unit, San Giuseppe Moscati Hospital, Road to Martina Franca, 74l 00 Taranto, Italy, Tel +39099 4585I80, Email lazzaigrazia@gmail.com

Received: July 12, 2020 | Published: August 04, 2020

\section{Introduction}

The incidence of nonmelanoma skin cancer (NMSC) is increasing worldwide annually and further increases can be expected due to population aging. ${ }^{1}$ Indeed, a recent systematic review reported that the very elderly ( $\geq 80$ years) population is rapidly growing worldwide, with an increasing incidence of NMSCs, ranging from 13 to 12112 per 100000 person-years, with lesions most frequently located in the head and neck region. ${ }^{2}$

Untreated suspected NMSC lesions in elderly patients must be carefully considered and promptly managed. These lesions show significantly heterogeneous growth patterns, with some untreated lesions growing rapidly and exhibiting aggressive features. ${ }^{3}$ Typical findings of untreated and neglected lesions in this population are the so-called giant NMSCs; by definition, giant NMSCs are lesions with diameter $\geq 5 \mathrm{~cm}$, including basal cell carcinoma (BCC) and squamous cell carcinoma (SCC) histological types, and are often associated with a significantly increased risk of complications and mortality. ${ }^{4}$

BCC and SCC account for $80 \%$ of NMSC lesions in the scalp or facial high-risk areas, referred to as the "Hzone" or "mask area". Among high risk factors for recurrence and metastases, both dimension $>2 \mathrm{~cm}$ and primary site, $\mathrm{H}$ zone are features indicative of aggressiveness, ${ }^{5}$ together with lesion thickness and neurovascular involvement.
Neglect of patients can be considered an additional risk factor, as it leads to delays in diagnosis and prompt treatment, given the tendency of these lesions to grow slowly. ${ }^{7}$ Moreover, frailty due to diabetes, vascular disorders, and advanced age must also be considered. In particular, aging skin appears to be predisposed to develop destructive lesions, as it undergoes progressive and degenerative changes of the supporting dermis, as well as vascular disorders, leading to decreased ability to repair skin damage. ${ }^{8}$ Poor hygiene, immunosuppression, and low socio-economic status also impact negatively on evolution of these lesions, even leading to myiasis in severe cases. ${ }^{9}$ Thus, neglected lesions in elderly patients can grow rapidly, become giant, and infiltrate the underlying tissues, with aggressive features ${ }^{10}$ such as inhomogeneous ulceration, hypertrophic and bleeding borders, tissue loss with deep scalp infiltration, or myiasis. ${ }^{11-13}$ Consequently, these lesions develop very uncomfortable features, which are particularly difficult to manage with aggressive reconstructive surgery. ${ }^{14}$ In this setting, electron beam 3D conformal radiotherapy (CRT), with conventional or hypo-fractionated modality, may be considered a good option for palliation or local control. Despite the high incidence of giant NMSCs in this demographic, little has been documented regarding its treatment with radiotherapy and outcomes, as these patients are underrepresented in the literature, ${ }^{15}$ and are not frequently referred to radiation oncologists. ${ }^{16}$ 
Here we report our retrospective experience of the feasibility and efficacy of electron beam 3D-CRT for the treatment of giant NMSC on scalp and skin $\mathrm{H}$ zone lesions in older and frail patients, with the aim of encouraging clinicians to offer an effective treatment, such as modern radiotherapy, to affected patients.

\section{Materials and methods}

Fifty older patients and a total of sixty measurable lesions were included in this analysis. Data were collected retrospectively on case report forms from the medical records of the patients. Older patients with giant and necrotic T3-T4 squamous skin lesions of the scalp and other regions of the $\mathrm{H}$ zone (central face, nasal, periocular, retroauricular), treated at San Giuseppe Moscati Radiotherapy Unit, Taranto, Italy, from 2005 through 2015, were included into this retrospective study. Written informed consent for the analysis of data was obtained from the patients and the Ethical Committee provided approval for the study. Pre-treatment biopsy of giant NMSCs had been performed for all patients, to confirm the diagnosis prior to treatment. Lesions were measured clinically using a ruler and documented with a series of digital photographs of each patient at baseline prior to treatment, then at one, three, and six months, and annually thereafter, to follow-up the clinical outcome.

\section{Patient and lesion characteristics}

Fifty consecutive very old and frail patients treated for inoperable giant T3-T4 high risk skin lesions of the scalp and other skin regions of the $\mathrm{H}$ zone were retrospectively studied. The patients were primarily male (30 males and 20 females), with a median age of 83 (range, 75-93) years. Sixty lesions were treated. Two synchronous adjacent lesions were recorded in five patients. There was a high proportion of tumors of the scalp (20 lesions, $33 \%$ ) and $45 \%$ of lesion with a diameter over $7 \mathrm{~cm}$. The mean maximum diameter of lesions was 8 (range, 6-12) cm.

Almost of lesions had deep tissue involvement with bleeding $(80 \%)$, and $30 \%$ exhibited purulent secretion. Most patients $(70 \%)$ described the lesions as painful. Of the 60 lesions, basal histology was determined in 40 and squamous histology in the remaining 20. Myiasis was detected in 2 patients. The Charlson Comorbidity index (CCI) $0-1$ was calculated, to account for diabetes and cardiac disease; ${ }^{17}$ CCI 1 was recorded in 40 patients (Table 1). The mean Karnofsky performance status was 70 (range, 60-100). Clinical characteristics are presented in Table 1.

Table I Baseline demographics, clinical and radiation treatment characteristics

\begin{tabular}{lll}
\hline Patients & $\mathbf{5 0}$ & $\mathbf{3 0 M} / \mathbf{2 0 F}$ \\
\hline Lesions & 60 & 20 scalp (33\%) \\
Mean age & $83 y r s$ & $75-93$ \\
Mean diameter & $8 \mathrm{~cm}$ & $6-12 \mathrm{~cm}$ \\
Diameter $>7 \mathrm{~cm}$ & 27 lesions & $45 \%$ \\
BBC & 40 lesions & $66 \%$ \\
SSC & 20 lesions & $33 \%$ \\
Bleeding & 48 lesions & $80 \%$ \\
Necrosis & 30 lesions & $50 \%$ \\
Pain & 35 pts & $70 \%$ \\
CCl I & 40 pts & $66 \%$ \\
\hline
\end{tabular}

Table Cotinued...

\begin{tabular}{lll}
\hline Patients & $\mathbf{5 0}$ & $\mathbf{3 0 M} / \mathbf{2 0 F}$ \\
\hline RT 60Gy /2Gy/fr & 27 lesions & $45 \%$ \\
RT 50Gy/ 2.5Gy/fr & 20 lesions & $33 \%$ \\
RT 48Gy/ 4Gy/fr & 13 lesions & $21 \%$ \\
KPS score 90-100\% & 15 pts & $30 \%$ \\
KPS score 70-80\% & 25 pts & $50 \%$ \\
KPS score 60\% & 10 pts & $20 \%$
\end{tabular}

yrs, years; pts, patients; KPS, karnofsky performance status; $\mathrm{CCl}$, comorbidity charlson index

\section{Radiation treatment}

Patient treatments were planned using Masterplan Treatment Planning ${ }^{\circledR}$ (Nucletron v.1/Elekta) and performed by 3D-CRT with a linear accelerator (Precise ${ }^{\circledR} /$ Elekta). Customized devices, using a thermoplastic mask (Civco ${ }^{\circledR}$, Orange City, IA) for scalp and facial lesions, were used to immobilize the head in supine or prone position and locate the area to be treated. The use of $3 \mathrm{D}$ conformal based planning was adopted as the lesions were large and deeply invading. A planning computed tomography (CT) scan, consisting of $2.5 \mathrm{~mm}$ thick slices of the head, from $2 \mathrm{~cm}$ in the air over the scalp to a line below the $\mathrm{C} 3$ vertebra, was performed to choose the electron energy, according to the depth of involvement and dose distribution. The findings on CT images corresponding to the visible surface lesion was defined as the gross tumor volume, and the lesion borders marked with plastic spherical $5 \mathrm{~mm}$-thick markers. An additional isotropic margin, 10 to $20 \mathrm{~mm}$ around the lesion, was added, to account for deep tissue involvement, defining the clinical target volume, according to histological type.$^{18} \mathrm{An}$ isotropic margin of $10 \mathrm{~mm}$ was provided, to obtain the planning target volume (PTV), to account for measurement uncertainty, electron profile edge effects, and uncertainty in customized shielding placement. Treatment consisted of one to two fields of customized electron beams; two adjacent matched electron angled beams were applied to treat large and irregular shaped, or two adjacent, lesions. ${ }^{19}$ Energies ranged from $8-15 \mathrm{MeV}$, with or without a customized bolus to increase the surface dose, and lead screen electron applicators that were large enough to encompass the entire PTV, according to International Commission on Radiation Units and Measurements recommendations. ${ }^{20}$ The total delivered dose varied depending on the target size and the treatment intent. For curative intent of lesions $>8 \mathrm{~cm}$ maximum diameter, $60 \mathrm{~Gy}$ in 30 fractions (2 $\mathrm{Gy} / \mathrm{fr}$ ) was advised; $50 \mathrm{~Gy}$ in 20 fractions $(2.5 \mathrm{~Gy} / \mathrm{fr})$ was prescribed for others, while in cases with bleeding or painful necrotic ulcers, extreme palliative radiotherapy, using $48 \mathrm{~Gy}$ in 12 fractions (4 Gy/fr) was delivered (Table 1)

\section{Endpoints and measurements}

Given the poor life expectancy of these patients, which could represent a bias in overall survival (OS) estimation, local progression free survival (LPFS) at 2 and 5 years were the main outcomes determined in this analysis, and were calculated starting from the end of radiotherapy through to time of relapse or clinical progression. Local relapse free survival was estimated as relapse of symptomatic lesion. OS was calculated starting from the end of radiotherapy through to death. Secondary end-points included investigation of the influence of clinical and dosimetric variables on local relapse survival, including: age, CCI, lesion size, primary site (scalp vs not scalp), deep tissue involvement, necrosis, electron energy, and radiation dose. In follow-up after completion of radiotherapy, patients were clinically 
evaluated one month later and every three months after radiotherapy during the first year, every six months for three years, and then annually. Clinical response was assessed as required by the response evaluation criteria in solid tumors (RECIST), version 1.1. ${ }^{21}$ Pain level was classified based on the Numeric Pain Rating Scale..$^{22}$ Toxicity was scored according to Radiation Therapy Oncology Group morbidity scoring criteria. ${ }^{23}$

\section{Statistical analysis}

Actuarial local progression-free survival at one and three years was estimated using the Kaplan-Meier method. Univariate analyses, with hazard ratio calculated by Chi-square and log-rank test, were used to detect statistical associations between clinical or dosimetric parameters and local progression. Pearson's covariance test was adopted for multivariate analyses. All statistical tests were two-sided and a p-value of $<0.05$ was considered to be statistically significant. Data were processed using SPSS Version 2.1, by normal license.

\section{Results}

\section{Survivals}

Median follow up from the treatment end was 5.5 (range, 3-8) years. Median OS was 56.8 months. OS rates at 2 and 5 years were $78 \%$ and $65 \%$, respectively. Comorbidities and advanced age were the major factors contributing to death.

\section{Local control}

Median LPFS was 2.8 years, while the cumulative LPFS rates at 2 and 5 years were $85 \%(95 \%$ CI $73-88)$ and $78 \%$ (95\% CI $68-80)$, respectively (Figure 1). Scalp lesion was significantly associated with durable local control, expressed as local relapse free survival (LRFS P $<0.02$, Kaplan Meier) (Figure 2).

On univariate analysis, several factors were found to significantly impact local progression, including: diabetes $(\mathrm{P}<0.001)$, age $>80$ years $(\mathrm{P}=0.034)$, lesion diameter $>7 \mathrm{~cm}(\mathrm{P}<0.046)$, necrosis $(\mathrm{P}=0.009)$, deep invasion $(\mathrm{P}=0.030)$, and scalp site $(\mathrm{P}<0.03)$ (Table 2$)$. No significant effects of fractionation or electron energy were detected. On Pearson's multivariate analysis, lesion diameter, necrosis, and primary site (not scalp vs scalp) were confirmed as the most significant factors indicating poor prognosis and inferior local control $(\mathrm{P}<0.001)$ (Table 2).

\section{Clinical response}

According RECIST, complete response (CR) occurred in 15 patients (30\%) and 25 lesions (41.6\%); partial response was achieved in 30 patients $(60 \%)$ and 27 lesions $(45 \%)$; and stable disease was observed in 5 patients $(10 \%)$ and 8 lesions $(13 \%)$. An example of $\mathrm{CR}$ in a 90 -year-old giant NMSC bearing patient after 60Gy electron beam radiotherapy is shown in Figure 2A-2B.

Table 2 Univariate cox proportional hazard ratio and multivariate analysis for local control

\begin{tabular}{|c|c|c|c|c|c|c|c|c|}
\hline \multirow{3}{*}{$\begin{array}{l}\text { Patients } 50 \text { (P) } \\
\text { Lesions } 60 \text { (L) }\end{array}$} & \multicolumn{6}{|c|}{ Univariate analysis } & \multicolumn{2}{|c|}{ Multivariate analysis } \\
\hline & \multicolumn{6}{|c|}{ Cox Prop HR } & \multicolumn{2}{|c|}{ Pearson's covariance } \\
\hline & $\mathbf{N}$ & HR & $95 \% \mathrm{Cl}$ & & $\mathbf{p}$ & $\mathbf{N}$ & $(95 \% \mathrm{Cl})$ & $\mathbf{p}$ \\
\hline \multicolumn{9}{|l|}{ Site $(\mathrm{L})$} \\
\hline scalp & 20 & ref & & & & 20 & & \\
\hline no scalp & 40 & 22 & $12-33$ & & $<0.003$ & 40 & $0.753^{* *}$ & $<0.001$ \\
\hline \multicolumn{9}{|l|}{ Diameter (L) } \\
\hline$<7 \mathrm{~cm}$ & 33 & ref & & & & 25 & & \\
\hline$>7 \mathrm{~cm}$ & 27 & 2.53 & I.0-6.45 & & 0.046 & 35 & $0.224 *$ & 0.03 \\
\hline \multicolumn{9}{|l|}{ Energy E- (L) } \\
\hline$E>12 \mathrm{MeV}$ & 40 & ref & & & & 40 & & \\
\hline $\mathrm{E}<12 \mathrm{MeV}$ & 20 & 0.65 & $0.3-1.9$ & & 0.382 & 20 & -0.083 & 0.38 \\
\hline \multicolumn{9}{|l|}{ Dose RT (L) } \\
\hline$<50 G y$ & 33 & ref & & & & 33 & & \\
\hline$>50 G y$ & 27 & 0.54 & $0.3-1.4$ & & 0.118 & 27 & -0.085 & 0.35 \\
\hline \multicolumn{9}{|l|}{ Necrosis (L) } \\
\hline yes & 30 & ref & & & & 10 & & \\
\hline no & 30 & 5.5 & $1.5-23.2$ & & 0.009 & 40 & $-0.54^{*}$ & 0.01 \\
\hline \multicolumn{9}{|l|}{ Deep Invasion (L) } \\
\hline yes & 35 & ref & & & & 35 & & \\
\hline no & 25 & 2.47 & $1.2-5.65$ & & 0.03 & 25 & $0.211 *$ & 0.03 \\
\hline \multicolumn{9}{|l|}{ N. Lesions $(P)$} \\
\hline 1 & 45 & ref & & & & 45 & & \\
\hline 2 & 5 & 1.7 & $0.7-3.93$ & 1.625 & 0.201 & 5 & -0.123 & 0.22 \\
\hline
\end{tabular}


Table Continued...

\begin{tabular}{|c|c|c|c|c|c|c|}
\hline Patients 50 (P) & Univariate analysis & & & & Multivar & analysis \\
\hline \multirow[t]{2}{*}{ Lesions 60 (L) } & Cox Prop HR & & & & Pearson' & variance \\
\hline & HR & $95 \% \mathrm{Cl}$ & $\mathbf{p}$ & $\mathbf{N}$ & (95\%Cl) & $\mathbf{p}$ \\
\hline
\end{tabular}

Diabetes $(\mathrm{P})$

yes

ref

no

35

18

I I-28

15

Age (P)

$<80 y r s$

20

ref

$0.3-1.53$

0.902

0.034

$\begin{array}{llllllllll}>80 y r s & 30 & 0.67 & 0.3-1.53 & 0.902 & 0.034 & 51 & -0.097 & 0.35 & \end{array}$

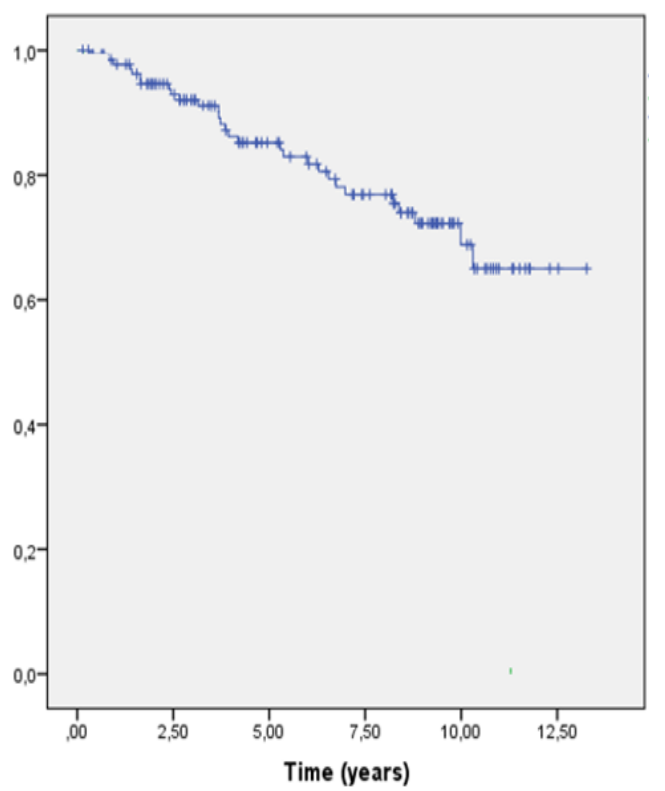

Figure I Kaplan-Meier overall local progression free survival (LPFS).



Figure 2 Kaplan - Meyer local relapse free survival (LRFS) between scalp (blue) vs no scalp (green) lesions.

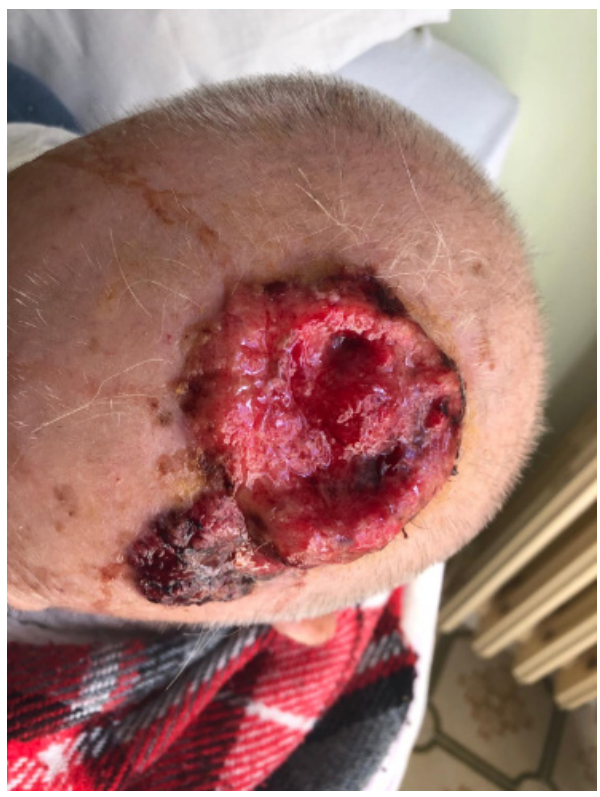

Figure $3 \mathrm{~A}$ case of giant NMSC of the scalp in a 93 years old patient measuring $10 \mathrm{~cm} \times 12 \mathrm{~cm}$ before $60 \mathrm{~Gy}$ electron beam radiotherapy.

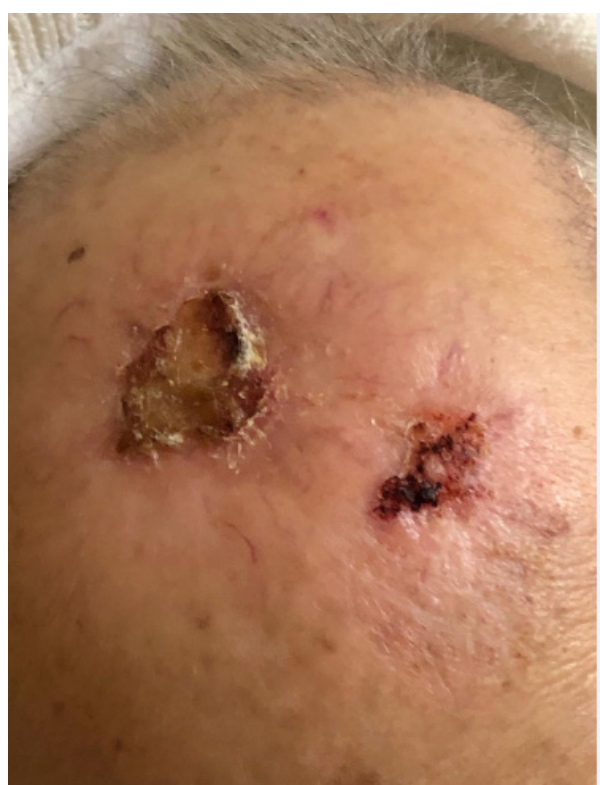

Figure 4 Same case 8 months later radiotherapy off showing a complete resolution of the lesion.

Citation: Lazzari G, Francesco P,Vittoria GS, et al. Electron beam radiotherapy in giant non- melanoma skin cancers of the head in elderly inoperable patients: a single centre retrospective study.J Cancer Prev Curr Res. 2020; I I (4):87-93. DOI: I0.15406/jcpcr.2020.I I.00434 
From a clinical perspective, bleeding control was obtained for all symptomatic lesions $(100 \%)$ and pain control was achieved for 25 symptomatic patients $(71 \%)$, with pain described as severe becoming moderate ( from 10 to 7,6 ) or mild (to $4-5$ ) in $90 \%$ of responding patients (Table 3).

\section{Toxicity}

The most important acute skin toxicities were G2, with erythema, desquamation, and edema in $80 \%$ of patients; G3 acute skin toxicity was recorded in $10 \%$ of cases with matched irradiated adjacent lesions. All cases required specific topical medication. G2 late skin toxicity occurred in $20 \%$ of patients, while G3 toxicity was observed in $6 \%$ of cases (Table 3 ).

Table 3 Results on clinical responses (RECIST criteria) and toxicity ( RTOG morbidity scoring criteria)

\begin{tabular}{ll}
\hline Complete response & I5 pts (30\%) - 25 Isn (4I.6\%) \\
Partial response & 30 pts (60\%) - 27 Isn (45\%) \\
Stable disease & 5 pts (I0\%) - 8 Isn (I3\%) \\
Bleeding control & 48 Isn (I00\%) \\
Pain control & $25 / 35$ pts (7I\%) \\
Acute skin toxicity G2/G3 & 40 pts $(80 \%) / 5 p t s(10 \%)$ \\
Late skin toxicity G2/G3 & 10 pts $(20 \%) / 3(6 \%)$ \\
\hline
\end{tabular}

pts, patients; Isn, lesions

\section{Discussion}

By definition, NMSCs with a diameter of $\geq 5 \mathrm{~cm}$ are considered giant, and exhibit features that fulfil the criteria for high-risk aggressive $\mathrm{BCC} / \mathrm{SCC} .{ }^{10}$ These tumors are usually localized to the scalp, mainly in older patients, and can show invasive features, causing extensive tissue ulceration, invading the periosteum, and subsequently eroding the underlying bone and extending into the cranial cavity, as occurs in scalp sites. ${ }^{24}$ Among therapies, surgery has been the main approach for decades..$^{25}$ Consequently, a great deal of evidence regarding complex surgical reconstructive management of these lesions has been published; ${ }^{26-28}$ however, reconstructive surgery can be extremely challenging in these cases, because of the requirement for complex surgical procedures, high risk American Society of Anesthesiologists (ASA) scores, and concurrent comorbidities in older patients, which can contraindicate this approach. Among sites, reconstruction of the scalp has been defined as a problematic procedure in older patients, because they have poor surrounding tissue which may be of insufficient quality for correction of large defects not amenable to primary or local flap closure. Thus, in this set of patients, megavoltage radiotherapy could offer an excellent option for care, as confirmed by a retrospective analysis of 108 patients with head and neck cancer with aggressive features, recording $87 \%$ actuarial 3-year local control and $82 \%$ relapse free survival. Radiotherapy has been assessed an effective primary treatment for NMSC ${ }^{16}$ with cure rates in most series exceeding $90 \%$, which is generally equivalent to surgery. ${ }^{29}$ Indeed, radiotherapy has an integral role in the treatment of BCC and SCC lesions of the head and neck in cosmetically sensitive areas, allowing for functional preservation of structures, where surgery could lead to functional impairment; however, as emphasized in a recent overview, ${ }^{16}$ this approach is underutilized in skin cancer care, despite published high-level evidence of its advantages, regardless of delivery modality, ${ }^{30}$ in terms of excellent local control rates in definitive settings. ${ }^{31}$ Further, the use of radiotherapy with palliative intent has been widely explored in elderly patients, providing excellent compliance with treatment, high response rates, symptom control, and improvements in quality of life, with toxicity profiles as acceptable as possible in frail patients, who are candidates for hypofractionated radiotherapy. ${ }^{32}$

Despite the growing evidence, little has been documented to date regarding the outcome of radiotherapy for giant NMSCs of the head skin and scalp. In a series of 20 giant tumors from a single center, only one patient underwent palliative radiotherapy, while two patients with high-risk scalp tumors were treated using adjuvant combined cetuximab/radiotherapy; however, clinical results are not available. ${ }^{4}$ Another report described a rare case of cutaneous myiasis located on a giant squamous cell carcinoma of the scalp in a 95 year old female who underwent radiation of 5,000-6,000 cGy in 24 daily fractions, without reporting of outcomes. ${ }^{33}$ To our knowledge, this retrospective study is the first to analyze the outcomes of giant NMSCs not amenable to surgery in a substantial group of older frail patients treated with definitive 3D CRT, demonstrating its feasibility and effectiveness in both curative and palliative settings.

In the past, experiences with external beam radiation modalities, including superficial X-rays $(45-100 \mathrm{kV})$, orthovoltage X-rays (100$250 \mathrm{kV}$ ), megavoltage photons, and electron beam radiation have been assessed in numerous retrospective studies, but on not selected skin lesions series. Consequently, local control ranging from $87 \%-100 \%$ at 2 to 5 years, with excellent to good cosmetic outcomes in the absence of grade 4 toxicities has been achieved. ${ }^{34,35}$ In particular, satisfactory therapeutic results have been obtained using superficial-X-ray therapy on head and neck NMSCs, as reported by Marconi et al. ${ }^{36}$ Further, high dose rate brachytherapy has been assessed as effective and convenient for treatment of NMSCs of the face, using surface molds with a radioisotope source (Ir-192) and hypofractionation delivery, providing a high local control rates (median, 97\%) and good cosmetic outcomes in $94.8 \%$ of cases, even in elderly patients. ${ }^{37}$ Similarly, electronic brachytherapy, based on a hypofractionation approach, can provide good local control rates and favorable cosmetic outcomes. ${ }^{38}$ But all these aforementioned techniques are inappropriate for treatment of giant head NMSCs, as they provide inadequate dosimetry in scenarios with deeper lesions $(>50 \mathrm{~mm})$, requiring image-based details of deep tissue infiltration and accurate margins, to improve target definition and dose coverage.

In recent years, the use of 3D conventional or IMRT electron beam radiotherapy, which can improve dosimetric issues by delineating target volumes in cases with large and deeply invading NMSCs, has increased. ${ }^{39}$ Modern electron beam radiotherapy is a feasible and versatile modality for treatment of giant, irregularly shaped skin lesions, with optimal dosimetric accuracy, where the properties of electrons provide a solution for cases with large or several adjacent lesions, as in our cases. Indeed, electron beams deliver high surface doses with a rapid dose falloff on defined skin targets, decreasing the radiation exposure of underlying brain tissues or lens. ${ }^{40}$ Thus, as reported here, megavoltage electron beam radiotherapy remains the most common choice to irradiate wide scalp lesions, as demonstrated in cases of total scalp irradiation using different technical solutions involving electron beam 3D-CRT. ${ }^{41}$ To overcome the inhomogeneous dose distribution disadvantage of electron beams, several combined volumetric arc therapy approaches have recently been tested, to obtain superior target coverage and produce concave or convex dose distributions, in agreement with the scalp conformation. ${ }^{42}$ Nevertheless, this modern treatment approach is chosen based on 
the available resources at institutions, thus electron beam 3D-CRT remains the most widely available treatment modality. The results of our experience using electron beam 3D-CRT for elderly frail patients with giant NMSCs of the head appear to be consistent with all previously published reports of managing NMSCs. ${ }^{30}$

The main limitation of the present study is its retrospective nature, however our findings agree with those identified in the current literature ${ }^{43}$ and our clinical management was completely in line with published guidelines which recommend the use of radiotherapy for older patients, anatomical sites not suitable for surgery, and where surgery is contraindicated. Further, the fractionation schedules chosen for each case were according to the guidelines, which suggest the adoption of conventional fractionation, with 20-30 fractions over four to six weeks in cases with very large lesions $(>6 \mathrm{~cm})$, as reported here. ${ }^{44}$

\section{Conclusion}

Giant NMSCs of the head are uncomfortable and not amenable to surgery in very old and frail patients. Radiotherapy with $3 \mathrm{D}$ conformal electron beam modality is a feasible and effective approach for local and symptom control, and provides excellent cosmetic results in this population, which is not frequently referred to radiation oncologists. Given its underutilization in this population, and the technological advances in this field, radiation oncologists should be increasingly asked to treat older patients with giant NMSCs of the head unsuitable for surgical intervention.

\section{Acknowledgments}

Statistical support was provided by Annalisa Digennaro, Statistician and Physician.

\section{Conflicts of interest}

The authors declare no conflict of interest.

\section{Funding}

No funding supported this study.

\section{References}

1. Jemal A, Siegel R, Ward E, et al. Cancer statistics, 2009. CA Cancer J Clin. 2009;59(4):225-249.

2. Lubeek SF, van Vugt LJ, Aben KK, et al. The epidemiology and clinicopathological features of basal cell carcinoma in patients 80 years and older : a systematic review. JAMA Dermatol. 2017;153(1):71-78.

3. Wehner MR, Dalma N, Landefeld C, et al. Natural history of lesions suspicious for basal cell carcinoma in older adults in Ikaria, Greece. $\mathrm{Br} J$ Dermatol. 2018;179(3):767-768.

4. Wollina U, Bayyoud Y, Kronert C, et al. Giant epithelial malignancies (Basal cell carcinoma, squamous cell carcinoma): a series of 20 tumors from a single center. J Cutan Aesthet Surg. 2012;5(1):12-19.

5. Eroglu A, Berberoglu U, Berreroglu S. Risk factors related to locoregional recurrence in squamous cell carcinoma of the skin. J Surg Oncol. 1996;61(2):124-130.

6. Haisma MS, Plaat BE, Bijl HP, et al. Multivariate analysis of potential risk factors for lymph node metastasis in patients with cutaneous squamous cell carcinoma of the head and neck. J Am Acad Dermatol. 2016;75(4):722-730.

7. Schofield JK, Linos E, Callander J. Management of skin cancer in the frail elderly: time for a rethink?. Br J Dermatol. 2016;175(5):855-856.
8. Farage MA, Miller KW, Berardesca E, et al. Clinical implications of aging skin: cutaneous disorders in the elderly. Am J Clin Dermatol. 2009;10(2):73-86.

9. Villwock JA, Harris TM. Head and neck myiasis, cutaneous malignancy, and infection: a case series and review of the literature. J Emerg Med. 2014;47(2):37-41.

10. Rishi A, Hui Huang S, O’Sullivan B, et al. Outcome following radiotherapy for head and neck basal cell carcinoma with aggressive features. Oral Oncol. 2017;72:157-164.

11. Selcuk G, Bilge KA, Ahmet E, et al. Intracranial Extension of Squamous Cell Carcinoma of the Scalp: A Case Report. J Neurol Sciences (Turkish). 2013;30(2):406-410.

12. Sesterhenn AM, Pfutzner W, Braulke DM, et al. Cutaneous manifestation of myiasis in malignant wounds of the head and neck. Eur J Dermatol. 2009;19(1):64-68.

13. Koyfman SA, Cooper JS, Beitler JJ, et al. ACR appropriateness criteria for aggressive nonmelanomatous skin cancer of the head and neck. Head Neck. 2016;38(2):175-182.

14. Lackey PL, Sargent LA, Wong L, et al. Giant basal cell carcinoma surgical management and reconstructive challenges. Ann Plast Surg. 2007;58(3):250-254.

15. Archontaki M, Stavrianos SD, Korkolis DP, et al. Giant basal cell carcinoma: Clinicopathological analysis of 51 cases and review of the literature. Anticancer Res. 2009;29(7):2655-2663.

16. Veness MJ, Delishaj D, Barnes EA, et al. Current role of radiotherapy in non-melanoma skin cancer. Clin Oncol. 2019;31(11):749-758.

17. Chaudhry S, Jin L, Meltzer D. Use of a self -report-generated Charlson Comorbidity index for predicting mortality. Med Care. 2005;43(6):607615.

18. Khan I, Choo R, Breen D, et al. Recommendations for CTV margins in radiotherapy planning for non- melanoma skin cancer. Radiother Oncol. 2012;104(2):263-266.

19. Bentel GC. Isodose Charts and Typical field arrangement. In: Radiation Therapy planning. Mac Grow Hill; 98-120.

20. International Commission on Radiation Units and Measurements. Prescribing, recording, and reporting electron beam therapy. ICRU Report 71. J ICRU. 2004:1(4).

21. Eisenhauer EA, Therasseb P, Bogaertsc J, et al. New response evaluation criteria in solid tumours: Revised RECIST guideline (version 1.1). Eur J Cancer. 2009;45(2):228-247.

22. McCaffery M, Beebe A. Pain: Clinical manual for nursing practice. Mosby St. Louis, MO; 1989.

23. Cox JD, Stetz J, Pajak TF. Toxicity criteria of the Radiation Therapy Oncology Group (RTOG) and the European Organization for Research and Treatment of Cancer (EORTC). Int J Radiat Oncol Biol Phys. 1995;31:1341-1346.

24. Zoccali G, Pajadan R, Papa P, et al. Giant basal cell carcinoma of the skin: Literature review and personal experience. J Eur Acad Dermatol Venereol. 2011;26(8):942-952.

25. Soma PF, Chibbaro S, Makiese O, et al. Aggressive scalp carcinoma with intracranial extension: a multidisciplinary experience of 25 patients with long-term follow-up. J Clin Neurosci. 2008;15(9):988-992.

26. Lackey PL, Sargent LA, Wong L, et al. Giant basal cell carcinoma surgical management and reconstructive challenges. Ann Plast Surg. 2007;58(3):250-254.

27. Wollina U, Bayyoud Y. Reconstruction of a large scalp defect by the sequential use of dermal substitute, self-filling osmotic tissue expander and rotational flap. J Cutan Aesthet Surg. 2010;3(2):106-110. 
28. Prera E, Pfeiffer J. Galea-aponeurotic flap for the repair of large scalp defects extending to bone. Auris Nasus Larynx. 2015;42(2):156-159.

29. Ducassou A, David I, Filleron T, et al. Retrospective analysis of local control and cosmetic outcome of 147 periorificial carcinomas of the face treated with low-dose rate interstitial brachytherapy. Int J Radiat Oncol Biol Phys. 2011;81(3):726-731.

30. Chua B, Jackson JE, Lin C, et al. Radiotherapy for early non-melanoma skin cancer. Oral Oncol. 2019;98:96-101.

31. Strom TJ, Caudell JJ, Harrison LB. Management of BCC and SCC of the Head and Neck. Cancer Control. 2016;23(3):220-227.

32. Porceddu SV, Rosser B, Burmeister BH, et al. Hypofractionated radiotherapy for the palliation of advanced head and neck cancer in patients unsuitable for curative treatment--"Hypo Trial". Radioter Oncol. 2007;85(3):456-462.

33. Biswasa S, McNerneya P. Myiasis on a Giant Squamous Cell Carcinoma of the Scalp: A Case Report and Review of Relevant Literature. World J Oncol. 2016;7(2-3):34-39.

34. Lovett RD, Perez CA, Shapiro SJ, et al. External irradiation of epithelial skin cancer. Int J Radiat Oncol Biol Phys. 1990;19(2):235-242.

35. Kwan W, Wilson D, Moravan V. Radiotherapy for locally advanced basal cell and squamous cell carcinomas of the skin. Int J Radiat Oncol Biol Phys. 2004;60(2):406-411.

36. Grossi Marconi D, da Costa Resende B, Rauber E, et al. Head and Neck Non-Melanoma Skin Cancer Treated By Superficial X-Ray Therapy: An Analysis of 1021 Cases. PLoS One. 2016;11(7):1-9.
37. Delishaj D, Rembielak A, Manfredi B et al. Non-melanoma skin cancer treated with high-dose-rate brachytherapy: a review of literature. $J$ Contemp Brachytherapy. 2016;8(6):533-540.

38. Bhatnagar A, Loper A. The initial experience of electronic brachytherapy for the treatment of non-melanoma skin cancer. Radiat Oncol. 2010;5:8794.

39. Matthiesen $\mathrm{C}$, Thompson JS, Forest $\mathrm{C}$, et al. The role of radiotherapy for T4 non-melanoma skin carcinoma. $J$ Med Imaging Radiat Oncol. 2011;55(4):407-416.

40. Wojcicka JB, Lasher DE, McAfee SS, et al. Dosimetric comparison of three different treatment techniques in extensive scalp lesion irradiation. Radiother Oncol. 2009;91(2):255-260.

41. Caivano R, Fiorentino A, Pedicini P, et al. A radiotherapy technique for palliative total scalp irradiation. Ann Palliat Med. 2015;4(1):35-38.

42. Noor M, Al-Ghamdi SM, Chantel C, et al. Customized double-shell immobilization device combined with VMAT radiation treatment of basosquamous cell carcinoma of the scalp. J Appl Clin Med Phys. 2019;20(2):84-93.

43. Schmults CD, Karia PS, Carter JB, et al. Factors predictive of recurrence and death from cutaneous squamous cell carcinoma: a 10-year, singleinstitution cohort study. JAMA Dermatol. 2013;149(5):541-547.

44. Newlands C, Currie R, Memon A, et al. Non-melanoma skin cancer: United Kingdom National Multidisciplinary Guidelines. J Laryngol Otol. 2016;130(S2):125-132. 